Chapman University

Chapman University Digital Commons

Economics Faculty Articles and Research

Economics

1997

\title{
Demand Fluctuations and Capacity Utilization Under Duopoly
}

\author{
J.J. Gabszewicz \\ Université Catholique de Louvain \\ Sougata Poddar \\ Chapman University, poddar@chapman.edu
}

Follow this and additional works at: https://digitalcommons.chapman.edu/economics_articles

Part of the Economic Theory Commons, and the Other Economics Commons

\section{Recommended Citation}

Gabszewicz, J., \& Poddar, S. (1997). Demand fluctuations and capacity utilization under duopoly. Economic Theory, 10(1), $131-146$. doi: $10.1007 / \mathrm{s} 001990050150$

This Article is brought to you for free and open access by the Economics at Chapman University Digital Commons. It has been accepted for inclusion in Economics Faculty Articles and Research by an authorized administrator of Chapman University Digital Commons. For more information, please contact laughtin@chapman.edu. 


\section{Demand Fluctuations and Capacity Utilization Under Duopoly}

\section{Comments}

This is a working version of an article accepted for publication in Economic Theory, volume 10, issue 1, in 1997 following peer review. This article may not exactly replicate the final published version. The final publication is available at Springer via DOI: 10.1007/s001990050150.

\section{Copyright}

Springer 


\title{
DEMAND FLUCTUATIONS AND \\ CAPACITY UTILIZATION UNDER DUOPOLY
}

\author{
J. J. Gabszewicz * and S. Poddar*
}

September 1995

\begin{abstract}
This paper studies the impact of uncertain demand on firms' capacity decisions when they operate in an oligopolistic enviromment. We define a two-stage game where firms choose capacity in the first stage without knowing which state of nature is going to realize, and output levels in the second, knowing which state is realized. We prove the existence of a symmetric subgame perfect equilibrium at which firms are in excess capacity compared with the capacity they would choose in the Cournot certainty equivalent game.
\end{abstract}

* CORE, Université catholique de Louvain.

We thank Y. Smeers for his helpful and interesting comments.

This text presents research results of the Belgian Program on Interuniversity Poles of Attraction initiated by the Belgian State, Prime Minister's Office, Science Policy Programming. The scientific responsibility is assumed by its authors. 


\section{Introduction}

This paper studies the impact of uncertain demand on firms' capacity decisions when they operate in an oligopolistic enviromment. The effect of uncertain demand fuctuations on expected profits. expected price and capacity in competitive, or monopolistic industries has been extensively analysed (s૯e Oi (1961), Sandmo (1971). Leland (1972), Drèze and Gabszewicz (1967), Smith (1969), Drèze and Sheshinski (1976)). To the best of our knowledge, no similar contributions have been devoted to the same problem, with assuming that demand fluctuations are faced by firms in a context of strategic interaction. ${ }^{1}$ This context implies that firms must adjust their capacity not only in view of meeting output demand levels varying across the states of Nature, but also for providing best output replies against the capacity and output strategies chosen by rival firms. In this random environment, firms play a game simultaneously against Nature and against their rivals. We assume however a kind of sequentiality in this intertwined game: when firms have to make their output decisions, they know which state the Nature has chosen; but they do not know it when they make their capacity choice. This situation lends itself to be formalized as a two-period sequential game in which firms choose capacity in the first stage (without knowing which state is going to realize), and output levels in the second (knowing which state is realized). In the second stage, firms play a Cournot game, conditional on the capacity levels decided in the first stage. If, between the first and the second stage, Nature has chosen a "boom" demand function, a firm with a low capacity level chosen in the first stage is unable to play in the second stage the Cournot output corresponding to the boom, but can at best, play a quantity equal to its chosen capacity. On the contrary, if Nature has chosen a "recession" demand function, a firm having chosen a high capacity level can play the Cournot output corresponding to recession, but remains with a costly idle capacity. A subgame perfect equilibrium of the two-stage game defined above must take simultaneously into account the strategic aspects of firms' behaviour and the cost considerations related to possible underutilization of capacity.

\footnotetext{
${ }^{1}$ A recent literature considers infinitely repeated price competition when the industry is submitted to exogenous demand shocks (see, in particular, Rotenberg, J. and G. Saloner (1986) and Kandori, M. (1901)). Even if these contributions are somewhat related to the present paper, they are formulated in the totally different approach of a supergame theoretic model. The purpose is also different since they study the impact of demand shocks on collusive behaviour of firms.
} 
Our model assumes that total demand varies linearly with output in each state of Nature, with a random intercept over the states. The industry consists of two firms, each with the same cost structure of the inelastic type. For this context, first we prove the existence of a synmetric subgame perfect equilibrium. Then, we fully characterize it, in terms of the capacity chosen at equilibrium and of the set of states at which firms decide, for reducing the cost of capacity, to choose a capacity smaller than the Cournot output level corresponding to these states.

Under pure competition, it is known that firms operate production, on average, at a level which is smaller than capacity. This is due to the fact that the number of firms for which expected profits are equal to zero, exceeds the number of firms for which the average cost of output per firm is minimum (see, for instance, Drèze and Sheshinski (1976)). Here we obtain an analogous excess capacity property when we compare the capacity chosen by the firms at the symmetric subgame perfect equilibrium, with the capacity they would choose if they would play a Cournot game with a "certainty equivalent" demand function. We found that uncertain capacity chosen by the firms at the symmetric subgame perfect equilibrium is at least greater than or equal to the capacity they would choose if they would play a Cournot game with "certainty equivalent" demand function. By contrast, expected output and expected price are shown to coincide at equilibrium with the output and price resulting from the same Cournot "certainty equivalent" game.

We present the model and the two-stage game in section 2. Section 3 is devoted to the existence and characterization of the symmetric subgame perfect equilibrium for the two-stage game defined in section 2. Section 4 compares the duopoly solution with the monopoly case, and with the "certainty equivalent" Cournot game. We end up with some concluding remarks collected in section 5 .

\section{The model}

We consider two firms with the same cost structure facing uncertain demand. The cost structure is of inelastic type: when production does not exceed capacity, costs increase linearly with output; when production exceeds capacity, costs are infinite, i.e.

$$
\begin{aligned}
C(k, q) & =\beta k+\gamma q, & & q \leq k \\
& =\infty, & & k<q .
\end{aligned}
$$


We assume that there are $n$ states of Nature and the demand in state $i$ is given by

$$
P_{i}(Q)=A_{i}-Q, \quad A_{i}<A_{i+1}, i=1, \ldots, n,
$$

with $Q$ denoting aggregate supply. Furthermore we assume that $A_{i}>\gamma+\beta$, $i=1, \cdots, n$.

We study a two-stage game in which firms choose capacity $k_{1}$ and $k_{2}$ in the first stage and output levels $q_{1}$ and $q_{2}$ in the second stage. When choosing capacity, firms do not know which state will realize, while they choose output knowing which state has realized. We assume the existence of an objective probability density $\rho_{i}$, $i=1, \ldots, n$, over the states of Nature, $\sum_{i=1}^{n} \rho_{i}=1$. Firms are assumed to be risk-neutral. We are interested in a symmetric subgame perfect equilibrium corresponding to firms' strategies $\left(k_{j}, q_{j}\right)_{j=1,2}$, in which firm $j$ chooses first capacity $k_{j}$ and then output level $q_{j}$.

Assume that firms have chosen capacities $k_{1}$ and $k_{2}$ in the first stage and let us consider the second stage game. The second stage payoff of firm $j$ in state $i$ is defined by

$$
\begin{aligned}
\Pi_{i j}\left(q_{i}, q_{h}, k_{j}, k_{h}\right) & =\left(A_{i}-q_{j}-q_{h}\right) q_{j}-\gamma q_{j}-\beta k_{j}, \text { if } q_{j} \leq k_{j} \text { and } q_{h} \leq k_{h} ; \\
& =\left(A_{i}-q_{j}-k_{h}\right) q_{j}-\gamma q_{j}-\beta k_{j}, \text { if } q_{j} \leq k_{j} \text { and } q_{h}>k_{h} ; \\
& =\left(A_{i}-k_{j}-k_{h}\right) k_{j}-\gamma k_{j}-\beta k_{j}, \text { if } q_{j}>k_{j} \text { and } q_{h}>k_{h} .
\end{aligned}
$$

It is easy to verify that, when both firms' capacities $k_{1}$ and $k_{2}$ exceed the Cournot output $\frac{A_{i}-\gamma}{3}$ in state $i$, the unique second stage "unconstrained" Nash equilibrium is given by $\left(\frac{A_{i}-\gamma}{3}, \frac{A_{i}-\gamma}{3}\right)$ in state $i$. When both firms' capacities $k_{1}$ and $k_{2}$ are smaller than the Cournot outcome $\frac{A_{i}-\gamma}{3}$ in state $i$, the unique second stage Nash equilibrium in state $i$ is given by $\left(k_{1}, k_{2}\right)$. In the asymmetric case, when $k_{j} \geq \frac{A_{i}-\gamma}{3}$ while $k_{h}<\frac{A_{i}-\gamma}{3}$, the unique second stage Nash equilibrium in state $i$ is $\left[\min \left\{k_{j}, \frac{A_{i}-k_{h}-\gamma}{2}\right\}, k_{h}\right]$ where $\frac{A_{i}-k_{h}-\gamma}{2}$ is the best reply output of firm $j$ against $k_{h}$.

Figures 1.1 and 1.2 illustrate the second stage equilibrium in state $i$. Figure 1.1 corresponds to the case where capacities $k_{1}$ and $k_{2}$ chosen in the first stage are both insufficient to produce the Cournot outcome $\left(\frac{A_{i}-\gamma}{3}, \frac{A_{i}-\gamma}{3}\right)$ in state $i$. Figure 1.2 illustrates the second stage equilibrium when firm 1 is capacity constrained at $k_{1}$ and firm 2 has chosen a capacity $k_{2}$ in the first stage sufficient to play its best reply against $k_{1}$. 


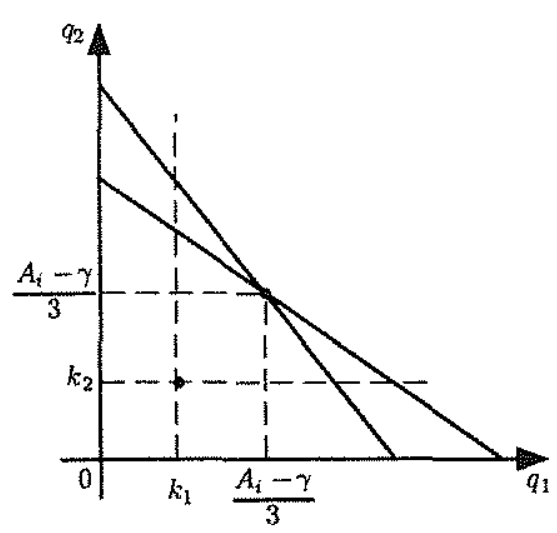

Figure 1.1

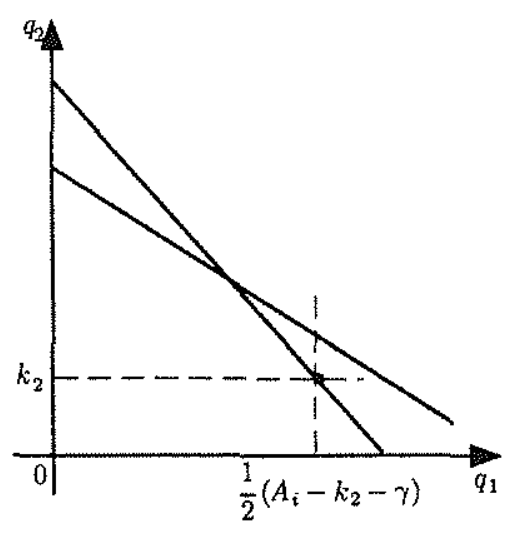

Figure 1.2

In the next section, we characterize a symmetric subgame perfect equilibrium (SSPE) and prove its existence. This symmetry allows us to define the first-stage game payoffs only on a restricted set of first stage strategies $\left(k_{1}, k_{2}\right)$.

\section{The symmetric subgame perfect equilibrium (SSPE)}

To characterize a SSPE, we shall write the first stage payoff of each firm under the assumption that both firms in the second stage are constrained (resp. unconstrained) by capacity on exactly the same subset of states of Nature. Indeed this property must necessarily hold at a SSPE whenever it exists: in such an equilibrium, both firms choose the same capacity level, which implies that unconstrained Cournot outcomes can be reached by both firms on exactly the same subset of states. Accordingly, in order to characterize a SSPE, we have only to consider the second stage equilibria which are given by $\left(k_{1}, k_{2}\right)$ in those states where capacity constraints are active, and by $\left(\frac{A_{i}-\gamma}{3}, \frac{A_{i}-\gamma}{3}\right)$ in those states $i$ where firms are not constrained by installed capacity. Since $A_{i}<A_{i+1}$ for all $i=1, \ldots, n$, it is clear that if capacities $\left(k_{1}, k_{2}\right)$ are binding for some state $s$ (namely, $k_{1}$ and $k_{2}$ are less than $\frac{A_{s}-\gamma}{3}$ ), they will be also binding for all states $i$, where $s<i<n$. 
Denoting by $i^{*}$ the largest index of states for which capacities are not binding. expected payoff $\pi_{i_{\mathrm{i}}}\left(k_{1}, k_{2}\right)$ for firm 1 is given by

$$
\Pi_{i_{1}^{*}}\left(k_{1}, k_{2}\right)=\sum_{s=1}^{i^{*}} \rho_{s}\left[\frac{\left(A_{s}-\gamma\right)^{2}}{9}-\beta k_{1}\right]+\sum_{s=i^{*}+1}^{n} \rho_{s}\left[\left(A_{s}-k_{1}-k_{2}-\gamma-\beta\right) k_{1}\right]
$$

and by $\Pi_{i \frac{*}{*}}\left(k_{1}, k_{2}\right)$, with

$$
\Pi_{i_{2}^{*}}\left(k_{1}, k_{2}\right)=\sum_{s=1}^{i^{*}} \rho_{s}\left[\frac{\left(A_{s}-\gamma\right)^{2}}{9}-\beta k_{2}\right]+\sum_{s=i^{*}+1}^{n} \rho_{s}\left[\left(A_{s}-k_{1}-k_{2}-\gamma-\beta\right) k_{2}\right]
$$

for firm 2 .

Consider a candidate SSPE of the two stage game, and let $i^{*}$ denote that state for which, at the corresponding candidate, both firms are not constrained up to state $i^{*}$, but constrained in the states $s$ with $i^{*}+1 \leq s \leq n$. At this candidate subgame perfect equilibrium, first-order conditions for a maximum should be satisfied, that is,

$$
\frac{\partial \Pi_{i_{1}^{*}}}{\partial k_{1}}=0 \Rightarrow \sum_{s=i^{*}+1}^{n} \rho_{s}\left[A_{s}-2 k_{1}-k_{2}-\gamma\right]-\beta=0
$$

and

$$
\frac{\partial \Pi_{i_{2}^{*}}}{\partial k_{2}}=0 \Rightarrow \sum_{s=i^{*}+1}^{n} \rho_{s}\left[A_{s}-2 k_{2}-k_{1}-\gamma\right]-\beta=0 .
$$

It is easy to verify that the second order conditions for a maximum are also satisfied. Now (4) holds $\Leftrightarrow$

$$
k_{1}=\frac{1}{2 \sum_{s=i^{*}+1}^{n} \rho_{s}}\left(\sum_{s=i^{*}+1}^{n} \rho_{s}\left(A_{s}-k_{2}-\gamma\right)\right)-\frac{\beta}{2 \sum_{s=i^{*}+1}^{n} \rho_{s}} .
$$

Similary, (5) holds $\Leftrightarrow$

$$
k_{2}=\frac{1}{2 \sum_{s=i^{*}+1}^{n} \rho_{s}}\left(\sum_{s=i^{*}+1}^{n} \rho_{s}\left(A_{s}-k_{1}-\gamma\right)\right)-\frac{\beta}{2 \sum_{s=i^{*}+1}^{n} \rho_{s}} .
$$


Using the symmetry property, we solve the above system for $k_{1}=k_{2}=k^{*}$, say, which yields

$$
k^{*}=\frac{1}{3}\left[\frac{\sum_{i^{*}+1}^{n} \rho_{s} A_{s}-\beta}{\sum_{i^{*}+1}^{n} \rho_{s}}-\gamma\right] .
$$

Notice in particular that, if it is optimal for the firm to choose $i^{*}+1$ to be equal to 1 , so that the firm chooses to be constrained in all states. then we get

$$
k^{*}=\frac{\sum_{s=1}^{n} \rho_{s} A_{s}-(\beta+\gamma)}{3} .
$$

Proposition 1. For the pair $\left(k^{*}, k^{*}\right)$ to be part of a SSPE with firms constrained from state $\left(i^{*}+1\right)$ onwards, it is necessarily true that

$$
\sum_{s>i^{*}+1} \rho_{s}\left(A_{s}-A_{i^{*}+1}<\beta \leq \sum_{s>i^{*}} \rho_{s}\left(A_{s}-A_{i^{*}}\right) .\right.
$$

Proof. To be a part of a SSPE with both firms unconstrained up to state $i^{*}, k^{*}$ must exceed the Cournot outcome under state $i^{*}$, i.e. $k^{*} \geq \frac{A_{i^{*}}-\gamma}{3}$, an inequality which is satisfied if, and only if, the right inequality of (8) holds. By a similar argument, it must be necessarily true that $k^{*}<\frac{A_{i^{*}+1}-\gamma}{3}$, an inequality which is satisfied if, and only if, the left inequality of (8) holds.

So far we have shown that, to be a part of a SSPE in which both firms are not capacity constrained up to state $i^{*}$, capacities must be defined by (6) and satisfy the parametric conditions (8). Yet, this is not sufficient to guarantee that $\left(k^{*}, k^{*}\right)$ is a SSPE. We have still to prove that, under $(8)$, no unilateral strategic deviation from $k^{*}$ can be advantageous to any firm.

Proposition 2. Under (8), the pair $\left(k^{*}, k^{*}\right)$ is part of a SSPE with firms constrained from state $\left(i^{*}+1\right)$ onwards.

Proof. See appendix for the detailed proof. Here we provide a sketch of the proof. Consider that firm 1 deviates from $k^{*}$ by increasing its capacity to a level $k_{1}$ which exceeds $k^{*}$, while firm 2 stays at $k^{*}$. As soon as $k_{1}$ exceeds the Cournot outcome 
in state $i^{*}+1$, firm 1 is no longer capacity-constrained in $i^{*}+1$; accordingly, the payoff in the first stage game as a function of $k_{1}$ is no longer provided by (2). From now onl. firm 1 can play its best reply against $k^{*}$ in all second stage games corresponding to states where these best replies are smaller or equal to $k_{1}$. Thus, given $k_{1}$, the first stage game payoff of firm 1 consists of three terms: the first term corresponds to the expected profit obtained from playing the Cournot outcome in all states $\sigma \leq i^{*}$; the second term corresponds to the expected profit of playing the best replies against $k^{*}$ in all second stage games corresponding to states where the best replies are smaller or equal to $k_{1}$. The third term represents the expected profit over all states where the capacity $k_{1}$ is not large enough to produce the best reply against $k^{*}$ in the corresponding states (the mathematical expression of this payoff is provided by formula (I) in the appendix). For instance, assume that $k_{1}$ belongs to the interval $\left[R_{i^{*}+1}\left(k^{*}\right), R_{i^{*}+2}\left(k^{*}\right)\right]$ where $R_{i^{*}+1}\left(k^{*}\right)$ (resp. $R_{i^{*}+2}\left(k^{*}\right)$ ) denotes the solution of the problem $\left.\max _{q}\left\{A_{i^{*}+1}-k^{*}-q\right) q-\gamma q\right\}$, (resp. $\left.\max _{q}\left\{A_{i^{*}+2}-k^{*}-q\right) q-\gamma q\right\}$ ), i.e. $R_{i^{*+1}}\left(k^{*}\right)$ (resp. $R_{i^{*}+2}\left(k^{*}\right)$ ) is the best reply against $k^{*}$ in the second-stage game in state $i^{*}+1$ (resp. $i^{*}+2$ ). Then the payoff $\Pi_{i^{*}+1}\left(k_{1}, k^{*}\right)$ for this deviation $k_{1}$ consists of the sum of three terms: (i) $\sum_{\sigma=1}^{i^{*}} \rho_{\sigma}\left[\left(\frac{A_{\sigma}-\gamma}{3}\right)^{2}-\beta k_{1}\right]$ (expected payoff of playing the Cournot outcome in states $\left.\sigma \leq i^{*}\right)$; (ii) $\rho_{i^{*}+1}\left\{\left[R_{i^{*}+1}\left(k^{*}\right)\right]^{2}-\beta k_{1}\right\}$ (expected payoff of playing the best reply against $k^{*}$ in the second stage game under state $\left.i^{*}+1\right)$; (iii) $\sum_{\sigma=i^{*}+2}^{n} \rho_{\sigma}\left(A_{\sigma}-\right.$ $\left.k^{*}-k_{1}-\gamma-\beta\right) k_{1}$ (expected payoff over the states where the firm is constrained to play $\left.k_{1}\right)$. It is shown in the appendix that the graph corresponding to this payoff can be represented as in figure 1, where the graph of the payoff $\Pi_{i^{*}}\left(k_{1}, k^{*}\right)$ is also plotted. The interesting fact is that the graph of $\Pi_{i^{*}+1}\left(k_{1}, k^{*}\right)$ is not only strictly concave, but it "cuts" the graph of $\Pi_{i} \cdot\left(k_{1}, k^{*}\right)$ when $k_{1}$ is equal to the best reply $R_{i^{*}+1}\left(k^{*}\right)$ in their respective decreasing parts. From this we conclude that the payoff $\Pi_{i^{*}+1}\left(k_{1}, k^{*}\right)$ for $k_{1} \in\left[R_{i^{*}+1}\left(k^{*}\right), R_{i^{*}+2}\left(k^{*}\right)[\right.$ is snaller than the payoff $\Pi_{i^{*}+1}\left(R_{i^{*}+1}\left(k^{*}\right), k^{*}\right)$. which itself is equal to $\Pi_{i^{*}}\left(R_{i^{*}+1}\left(k^{*}\right), k^{*}\right)$. Furthermore, we know that $\Pi_{i^{*}}\left(R_{i^{*}+1}\left(k^{*}\right), k^{*}\right)$ is less than $\Pi_{i^{*}}\left(k^{*}, k^{*}\right)$. Hence, no deviation $k_{1}$ in $\left[R_{i^{*}+1}\left(k^{*}\right), R_{i^{*}+2}\left(k^{*}\right)\right\}$ can be profitable. Then proceeding recursively by assuming $k_{1}$ in $\left[R_{i^{*}+2}\left(k^{*}\right), R_{i^{*}+3}\left(k^{*}\right)\left[, \cdots,\left[R_{n-1}\left(k^{*}\right), R_{n}\left(k^{*}\right)\right]\right.\right.$, we show that no deviation $k_{1} \geq k^{*}$ can be profitable for firm 1 . 


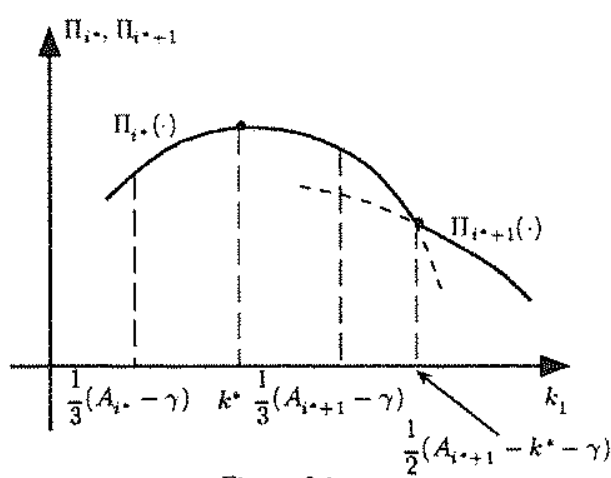

Figure 2.1

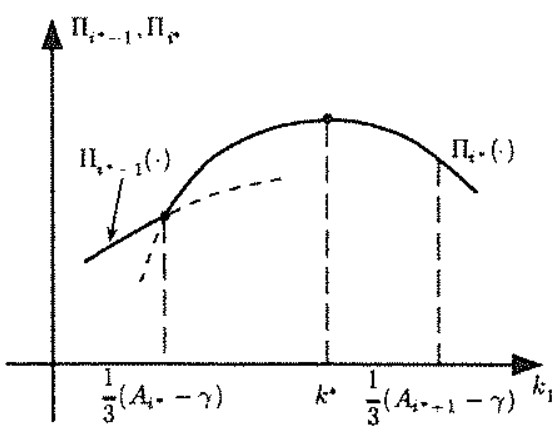

Figure 2.2

Now, assume that firm 1 deviates by decreasing its capacity from $k^{*}$, while firm 2 stays at $k^{*}$. Clearly no deviation $k_{1}$ with $\frac{A_{i^{*}}-\gamma}{3} \leq k_{1}<k^{*}$ can be profitable. So we must only consider deviations $k_{1}$ with $k_{1}<\frac{A_{i^{*}}-\gamma}{3}$. When $k_{1}$ is chosen between the Cournot outcomes in the second stage games under state $s$ and state $s+1,1<s \leq i^{*}-1$, the payoff of firm $1 \Pi_{s}\left(k_{1}, k^{*}\right)$ in the first stage game consists again in the sum of three terms (see formula (II) in the appendix): (i) $\sum_{\sigma=1}^{s-1} \rho_{\sigma}\left[\left(\frac{A_{\sigma}-\gamma}{3}\right)^{2}-\beta k_{1}\right]$ (expected payoff of playing the Cournot outcome in states $\sigma \leq s-1$ ); (ii) $\sum_{\sigma=s}^{i^{*}} \rho_{\sigma}\left[\left(A_{\sigma}-\frac{A_{\sigma}-\gamma}{3}-k_{1}-\gamma-\beta\right) k_{1}\right]$ (expected payoff in all states with firm 1 constrained by $k_{1}$ and firm 2 playing the corresponding Cournot outcome: remember that firm 2 has capacity equal to $k^{*}$, and can thus produce the Cournot outcome for all states $\sigma$ with $\sigma \leq i^{*}$ ); and (iii) $\sum_{\sigma=i^{*}+1}^{n} \rho_{\sigma}\left[\left(A_{\sigma}-k^{*}-k_{1}-\gamma-\beta\right) k_{1}\right]$ (expected payoff with both firms constrained, firm 1 by $k_{1}$, firm 2 by $k^{*}$ ). For instance, assume that $k_{1}$ is chosen in the interval $] \frac{A_{i^{*}-1}-\gamma}{3}, \frac{A_{i^{*}}-\gamma}{3}$ [ (i.e. $s=i^{*}-1$ ). Then the payoff $\Pi_{i^{*}-1}\left(k_{1}, k^{*}\right)$ in the first stage game obtains as

$$
\begin{aligned}
\Pi_{i^{*}-1}\left(k_{1}, k\right) & =\sum_{\sigma=1}^{i^{*}-1}\left(\frac{A_{\sigma}-\gamma}{3}\right)^{2}+\rho_{i^{*}}\left[\left(A_{i^{*}}-\frac{A_{i^{*}}-\gamma}{3}-k_{1}-\gamma\right) k_{1}\right] \\
& +\sum_{\sigma=i^{*}+1}^{n} \rho_{\sigma}\left(A_{\sigma}-k^{*}-k_{1}-\gamma\right) k_{1}-\beta k_{1} .
\end{aligned}
$$

It is shown in the appendix that the graph of $\Pi_{i^{*}-1}\left(k_{1}, k^{*}\right)$ can be represented as on figure 2 where the graph of the payoff $\Pi_{i^{*}}\left(k_{1}, k^{*}\right)$ is also plotted. The interesting fact is that not only the grapl of $\Pi_{i^{*}-1}\left(k_{1}, k^{*}\right)$ is strictly concave, but it "cuts" 
the graph of $\Pi_{i^{*}}\left(k_{1}, k^{*}\right)$ when $k_{1}$ is equal to the Cournot outcome $\frac{A_{i^{*}}-\gamma}{3}$ under state $i^{*}$ in their respective increasing parts. From this we conclude that the payoff $\Pi_{i^{*-1}}\left(k_{1}, k^{*}\right)$ is smaller than the payoff $\Pi_{i^{*-1}}\left(\frac{A_{i^{*}}-\gamma}{3}, k^{*}\right)$ which itself being equal to $\Pi_{i^{*}}\left(\frac{A_{i^{*}}-\gamma}{3}, k^{*}\right)$, is smaller than $\Pi_{i^{*}}\left(k^{*}, k^{*}\right)$. Accordingly, no deviation $k_{1}$ in the interval $\left[\frac{A_{i^{*}-1}-\gamma}{3}, \frac{A_{i^{*}}-\gamma}{3}[\right.$ can be profitable. Then proceeding recursively by assuming $k_{1}$ in $\left[\frac{A_{i^{*}-2}-\gamma}{3}, \frac{A_{i^{*}-1}-\gamma}{3}\left[\cdots,\left[\frac{A_{1}-\gamma}{3}, \frac{A_{2}-\gamma}{3}[\right.\right.\right.$, we show that no deviation $k_{1} \leq k^{*}$ can be profitable for firm 1. Applying a similar argument for deviations of firm 2 leads to a complete proof of proposition 2 .

\section{A comparison with monopoly and with the uncertainty equivalent case}

It is interesting to compare the duopoly solution which as just been derived with the monopoly case. Given state $i$, the monopolist's optimal output is $\frac{A_{i}-\gamma}{2}$. As under duopoly, the monopolist, facing the same uncertain demand and the same cost conditions, can freely choose its capacity and, accordingly, the subset of states under which this capacity exceeds the monopoly output in the corresponding states. If the monopolist's capacity $k_{m}$ is chosen so as to be unconstrained up to state $i_{m}$, expected profit writes as

$$
E\left(\Pi_{m}\left(k_{m}\right)\right)=\sum_{s=1}^{i_{m}} \rho_{s}\left[\left(\frac{A_{s}-\gamma}{2}\right)^{2}-\beta k_{m}\right]+\sum_{s=i_{m}+1}^{n} \rho_{s}\left(A_{s}-k_{m}-\gamma-\beta\right) k_{m} .
$$

The first order condition for a maximum requires that

$$
\frac{d E}{d k_{m}}=0 \Rightarrow \sum_{s=i_{m}+1}^{n} \rho_{s}\left(A_{s}-2 k_{m}-\gamma\right)=\beta
$$

Solving for $k_{m} \stackrel{\text { def }}{=} k_{m}^{*}$, leads to

$$
k_{m}^{*}=\frac{1}{2}\left[\frac{\sum_{s=i_{m}+1}^{n} \rho_{s} A_{s}-\beta}{\sum_{s=i_{m}+1}^{n} \rho_{s}}-\gamma\right] .
$$

An argument similar to the one used in the duopoly case shows that exactly the same condition on $\beta$ (see equation (8)) as in the duopoly case, guarantees that 
it is indeed optimal for the monopolist to choose $k_{m}$ so as to be unconstrained up to state $i_{m}$. However, a direct comparison between (9) and (6) reveals that $k_{m}^{*}<2 k^{*}$, so that total installed capacity in the duopoly case exceeds the installed capacity under monopoly.

Now, it is also of interest to compare both monopoly and duopoly capacities with the certainty equivalent case. This case is defined as a deterministic market, in which the demand function $\bar{P}(Q)$ would be equal to the expected demand function resulting from the randomness of demand over the $n$ states of Nature, i.e.

$$
\bar{P}(Q)=\sum_{i=1}^{n} \rho_{i} P_{i}(Q)=\sum_{i=1}^{n} \rho_{i}\left(A_{i}-Q\right)=\sum_{i=1}^{n} \rho_{i} A_{i} \sim Q
$$

with $Q$ denoting aggregate supply.

In the duopoly situation, it is easy to check that the Cournot outcome corresponding to the certainty equivalent case is given by

$$
\hat{k}_{1}=\hat{k}_{2}=\hat{q}_{1}=\hat{q}_{2}=\frac{\sum_{i=1}^{n} \rho_{i} A_{i}-(\beta+\gamma)}{3} \stackrel{\text { def }}{=} \hat{k},
$$

so that it coincides with the duopoly solution $k_{1}^{*}=k_{2}^{*}=k^{*}$ under uncertainty when firms choose to be constrained over all states (see ( $\gamma)$ ). In all other cases, when it is optimal for the firms to be constrained over a restricted set of states only, we get that $k^{*}>\hat{k}$, with $k^{*}$ defined by (6). Indeed. assume on the contrary that $k^{*} \leq \hat{k}$, with both firms having chosen to be constrained from state $i^{*}+1$ onwards. This implies that

$$
\beta>\sum_{s=1}^{n} \rho_{s} A_{s}-\frac{\sum_{s=1}^{i^{*}} \rho_{s} A_{s}}{\sum_{s=1}^{i^{*}} \rho_{s}} .
$$

Using the right hand side of (8), which holds since it is optimal for the firms to be constrained from state $i^{*}+1$ onwards, we get

$$
\beta \leq \sum_{s=i^{*}+1}^{n} \rho_{s}\left(A_{s}-A_{i^{*}}\right)
$$


Thus, combining (11) and (12), we must have

$$
\sum_{s=1}^{n} \rho_{s} A_{s}-\frac{\sum_{s=1}^{i^{*}} \rho_{s} A_{s}}{\sum_{s=1}^{i^{*}} \rho_{s}} \leq \sum_{s=i^{*}+1}^{n} \rho_{s}\left(A_{s}-A_{i^{*}}\right),
$$

which implies

$$
A_{i} \cdot\left(\sum_{s=1}^{i^{*}} \rho_{s}\right)<\sum_{s=1}^{i^{*}} \rho_{s} A_{s},
$$

a contradiction. Thus we have proved the following

Proposition 3. Whenever at the SSPE, firms choose to be unconstrained in at least one state, then the capacity chosen by the firms at equilibrium exceeds the capacity they would choose in the certainty equivalent case.

At the monopoly, the certainty equivalent optimal capacity $\hat{k}_{m}$ obtains as

$$
\hat{k}_{m}=\frac{1}{2}\left[\sum_{i=1}^{n} \rho_{i} A_{i}-(\beta+\gamma)\right] .
$$

A similar argument as in proposition 3 shows that, whenever it is optimal for the monopolist to be unconstrained in a least one state, then monopolist's optimal capacity $k_{m}^{*}$ exceeds $\hat{k}_{m}$.

Finally, it is worthwhile to perform some further comparisons. First, notice that market excess capacity: obtained by aggregating firms excess capacities as referred in proposition 3 , is greater under duopoly than under monopoly. Indeed, market excess capacity in the monopoly case is equal to $k_{m}^{*}-\hat{k}_{m}$. As under duopoly, market excess capacity is equal to $2\left(k^{*}-\hat{k}\right)$, and a direct comparison using (9), (13), (6) and (10), reveals that $k_{m}^{*}-\hat{k}_{m}<2\left(k^{*}-\hat{k}\right)$.

On the other hand, it is useful to compare, for the duopoly situation, the expected price and output in the uncertain demand case with price and output in the certainty equivalent case. For the latter, the output obtains as

$$
2 \hat{k}=\frac{2}{3}\left[\sum_{i=1}^{n} \rho_{i} A_{i}-(\beta+\gamma)\right]
$$


and price as

$$
\bar{P}(2 \hat{k})=\frac{\sum_{i=1}^{n} \rho_{i} A_{i}}{3}+\frac{2}{3}(\beta+\gamma) .
$$

In the uncertain demand case, under conditions (8) which guarantee that the firms choose the SSPE to be unconstrained up to state $i^{*}$, expected output writes as

$$
\frac{2}{3} \sum_{s=1}^{i^{*}} \rho_{s}\left(A_{s}-\gamma\right)+2 k^{*} \sum_{s=i^{*}+1}^{n} \rho_{s}
$$

which, by $(6)$, is equal to

$$
\frac{2}{3}\left[\sum_{s=1}^{n} \rho_{s} A_{s}-(\beta+\gamma)\right],
$$

as in (14). As for the expected price, we get

$$
\sum_{s=1}^{i^{*}} \rho_{s}\left(\frac{A_{s}+2 \gamma}{3}\right)+\sum_{s=i^{*}+1}^{n}\left(A_{s}-2 k^{*}\right)
$$

and it is easily checked that this expected price is equal to $\bar{P}(2 \bar{k})$. Accordingly, we obtain the following

Proposition 4. Whenever it is optimal for the firms to be unconstrained up so state $i^{*}$, expected price and output at the SSPE coincide with the price and output. corresponding to the certainty equivalent case.

\section{Concluding remarks}

In this paper we have analyzed the investment behaviour of firms when they are simultaneously faced with a random demand and with rival firms in the industry. Under the restricted assumption of a linear demand with a random intercept over the states of Nature, we have shown that there exists a symmetric equilibrium at which firms operate in excess capacity, when compared with the capacity they would choose in the certainty equivalent case. Our analysis is original, we feel, to this extent that it deals with the strategic behaviour of firms when they operate in an uncertain environment.

The paper calls for two natural extensions. The first would be to generalize the above analysis to other cost structures and different random demand environments. 
The other one would consider the problem of investment in entry deterrence when demand fluctuates randomly. We have in mind an extension of the paper by Dixit (1980) which would consider sequential entry in an industry faced with uncertain demand.

\section{References}

Dixit, A. (1980). The role of investment in entry deterrence. Economic Journal, 90, 95-106.

Drèze, J.H. and J.J. Gabszewicz (1967). Demand and fluctuations, capacity utilization and prices. Operations Research Verfahren, 3, 119-141.

Drèze, J.H. and E. Sheshinski (1976). Demand fluctuations, capacity utilization and costs. American Economic Review, 66, 731-742.

Kandori, M. (1991). Correlated demand shocks and price wars during booms. Review of Economic Studies, 58, 171-180.

Leland, H. (1972). Theory of the firm facing random demand. American Economic Review, 62, 278-291.

Oi, W. (1961). Uncertainty, prediction and competitive equilibrium. Quarterly Journal of Economics, 29, 58-64.

Rotemberg, J. and G. Saloner (1986). A supergame-theoretic model of price wars during booms. American Economic Review, 70, 390-407.

Sandmo, A. (1971). On the theory of the competitive firm under price uncertainty. American Economic Review, 61, 65-73.

Smith, K.R. (1969). The effect of uncertainty on monopoly price, capital stock and utilization of capital. Joumal of Esonomic Theory, 1, 48-59. 


\section{Appendix: Proof of Proposition 2}

If capacity is not binding under state $s$, the best reply against $k^{*}$ in the second-stage game obtains as the solution of the maximization problem

$$
\max _{q}\left(A_{s}-k^{*}-q\right) q-\gamma q
$$

i.e.

$$
\frac{A_{s}-k^{*}-\gamma}{2} \stackrel{\text { def }}{=} R_{s}\left(k^{*}\right)
$$

Now assume that firm 1 deviates from $k^{*}$ to some new level of capacity $k_{1} \geq k^{*}$. When $k^{*}<k_{1} \leq \frac{A_{i+1}-\gamma}{3}$ it is clear that such a deviation cannot be profitable since $k^{*}$ is the best reply in the first stage game against $k^{*}$ as long as $k_{1}$ is in the interval $\left[\frac{A_{i}-\gamma}{3}, \frac{A_{i+1}-\gamma}{3}\right]$. So we bave only to consider in the sequel values of $k_{1} \geq \frac{A_{i+1}-\gamma}{3}$. Let $s$ be any state with $i^{*}<s<n$. If $k_{1} \in\left[R_{s}\left(k^{*}\right), R_{s+1}\left(k^{*}\right)\right]^{\text {def }}=$ $I_{s}$, it is optimal for firm 1 to play $R_{\sigma}\left(k^{*}\right)$ in the second stage game in state $\sigma$, for $\sigma \in\left\{i^{*}+1, \cdots, s\right\}$. For $\sigma \in\{s+1, \cdots, n\}$, firm 1 is constrained by $k_{1}$ since it cannot use $R_{\sigma}\left(k^{*}\right)$ in the second stage game. Accordingly, the payoffs of firm 1 in the first stage game when $k_{2} \in\left[R_{s}\left(k^{*}\right), R_{s+1}\left(k^{*}\right)[\right.$ is given by:

$$
\begin{aligned}
\Pi_{s}\left(k_{1}, k^{*}\right) & \stackrel{\text { def }}{=} \sum_{\sigma=1}^{i^{*}} \rho_{\sigma}\left(\frac{A_{\sigma}-\gamma}{3}\right)^{2}+\sum_{\sigma=i^{*}+1}^{s} \rho_{\sigma}\left(\frac{A_{\sigma}-k^{*}-\gamma}{2}\right)^{2} \\
& +\sum_{\sigma=s+1}^{n} \rho_{\sigma}\left(A_{\sigma}-k_{1}-k^{*}-\gamma\right) k_{1}-\beta k_{1}
\end{aligned}
$$

Assume first that $k_{1} \in\left[\frac{A_{i^{*}+1}}{3}, \frac{A_{i^{*}+1}-k^{*}-\gamma}{2}[\right.$. Then payoffs to firm 1 of the first stage game is, given by (see (2))

$$
\Pi_{i^{*}}\left(k_{1}, k^{*}\right)=\sum_{\sigma=1}^{i^{*}} \rho_{\sigma}\left(\frac{A_{\sigma}-\gamma}{3}\right)^{2}+\sum_{\sigma=i^{*}+1}^{n} \rho_{\sigma}\left(A_{\sigma}-k_{1}-k^{*}-\gamma\right) k_{1}-\beta k_{1} .
$$

We know that $k_{1}=k^{*}$ is the best reply against $k^{*}$ for $k_{1}$ in this domain since the payoff (A.1) coincides with (2), from which $k^{*}$ has been shown to be the unique solution to the maximization problem.

Now assume that $k_{1} \in\left[\frac{A_{i^{*}+1}-k^{*}-\gamma}{2}, \frac{A_{i^{*}+2}-k^{*}-\gamma}{2}\right]=I_{i^{*}+1}$. In this domain the payoff of the first stage game given firm 2 plays $k^{*}$, obtains by applying 
formula (I) with $s=i^{*}+1$, i.e.

$$
\begin{aligned}
\Pi_{i^{*}+1}\left(k_{1}, k^{*}\right) & =\sum_{\sigma=1}^{i^{*}} \rho_{\sigma}\left(\frac{A_{\sigma}-\gamma}{3}\right)^{2}+\rho_{i^{*}+1}\left(\frac{A_{i^{*}+1}-k^{*}-\gamma}{2}\right)^{2} \\
& +\sum_{\sigma=i^{*}+2}^{n} \rho_{\sigma}\left(A_{\sigma}-k^{*}-k_{1}-\gamma\right) k_{1}-\beta k_{1} .
\end{aligned}
$$

It is easy to check that $\frac{\partial^{2} \Pi_{i^{*}+1}\left(k_{1}, k^{*}\right)}{\partial k_{1}^{2}}<0$, so that $\Pi_{i^{*}+1}$ is strictly concave. On the other hand,

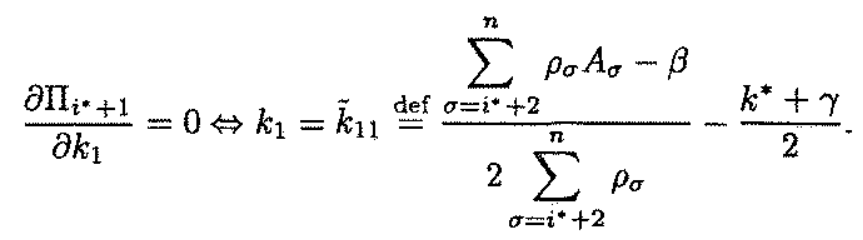

Now assume that

$$
\tilde{k}_{11} \geq \frac{A_{i^{*}+1}-k^{*}-\gamma}{2}
$$

This implies that

$$
\beta<\sum_{\sigma>i^{*}+1} \rho_{\sigma}\left(A_{\sigma}-A_{i^{*}+1}\right)
$$

which contradicts the left part of inequality (8), so that

$$
\tilde{k}_{11}<\frac{A_{i \cdot+1}-k^{*}-\gamma}{2}
$$

Accordingly, the value of $k_{1}$ which maximizes the function $\Pi_{i^{*}+1}\left(k_{1}, k^{*}\right)$ does not belong to $I_{i^{*}+1}$. Thus, by strict concavity of $\Pi_{i^{*}+1}\left(k_{1}, k^{*}\right)$, the optimal value of $\Pi_{i^{*}+1}$, when $k_{1}$ is restricted to $I_{i^{*}+2}$, lies on the left boundary of $I_{i^{*}+1}$, i.e. at $k_{1}=\frac{A_{i^{*}+1}-k^{*}-\gamma}{2}$. Moreover, substituting the values of $k_{1}$ in $(\mathrm{A} .1)$ in $\Pi_{i^{*}}\left(\cdot, k^{*}\right)$, we see that

$$
\Pi_{i^{*}}\left(\frac{A_{i^{*}+1}-k^{*}-\gamma}{2}, k^{*}\right)=\Pi_{i^{*}+1}\left(\frac{A_{i^{*}+1}-k^{*}-\gamma}{2}, k^{*}\right) .
$$

Since

$$
\Pi_{i^{*}}\left(\frac{A_{i^{*}+1}-k^{*}-\gamma}{2}, k^{*}\right)<\Pi_{i^{*}}\left(k^{*}, k^{*}\right)
$$

no deviation $k_{1}$ in $I_{i^{*}+1}$ is profitable. 
Now assume that $k_{1} \in I_{i^{*}+2}$. Applying fromula (I) with $s=i^{*}+2$, we get. for $k_{1} \in I_{i^{*}+2}$,

$$
\begin{aligned}
\Pi_{i^{*}+2}\left(k_{1}, k^{*}\right) & =\sum_{\sigma=1}^{i^{*}} \rho_{\sigma}\left(\frac{A_{\sigma}-\gamma}{3}\right)^{2}+\rho_{i^{*}+1}\left(\frac{A_{i^{*}+1}-k^{*}-\gamma}{2}\right)^{2} \\
& +\rho_{i^{*}+2}\left(\frac{A_{i^{*}+2}-k^{*}-\gamma}{2}\right)^{2} \sum_{\sigma=i^{*}+3}^{n} \rho_{\sigma}\left(A_{\sigma}-k^{*}-k_{1}-\gamma\right) k_{1}-\beta k_{1}
\end{aligned}
$$

It is easy to check that $\frac{\partial^{2} \Pi_{i^{*}+2}\left(k_{1}, k^{*}\right)}{\partial k_{1}^{2}}<0$, so that $\Pi_{i^{*}+2}$ is strictly concave. Furthermore,

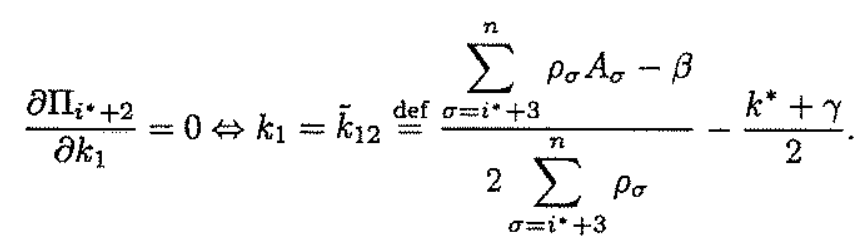

Now suppose that $\tilde{k}_{12} \geq \frac{A_{i^{*}+2}-k^{*}-\gamma}{2}$. This implies

$$
\beta \leq \sum_{\sigma=i^{*}+3}^{n}\left(A_{\sigma}-A_{i^{*}+2}\right)
$$

and, a fortiori,

$$
\beta \leq \sum_{\sigma=i^{*}+3}^{n} \rho_{\sigma}\left(A_{\sigma}-A_{i^{*}+2}\right)+\rho_{i^{*}+2}\left(A_{i^{*}+2}-A_{i^{*}+1}\right) .
$$

This, in turn, implies

$$
\beta \leq \sum_{\sigma=i^{*}+2}^{n} \rho_{\sigma}\left(A_{\sigma}-A_{i^{*}+1}\right)
$$

since $A_{i^{*}+2}>A_{i^{*}+1}$.

However the last inequality contradicts the left part of (A.1). Accordingly; the value of $k$, which maximizes $\Pi_{i^{*}+2}\left(k_{1}, k^{*}\right)$ does not belong to $I_{i^{*}+2}$. Thus, by strict concavity of $\Pi_{i^{+}+2}$, the optimal value of $\Pi_{i^{*}+2}$, when $k_{1}$ is restricted to $I_{i^{*}+2}$, lies on the left boundary of $I_{i^{*}+2}$, i.e at $k_{1}=\frac{A_{i^{*}+2}-k^{*}-\gamma}{2}$. Hence, for all $k_{1} \in I_{i^{+}+2}^{*}$, we have

$$
\Pi_{i^{*}+2}\left(k_{1}, k^{*}\right) \leq \pi_{i^{*}+2}\left(\frac{A_{i^{*}+2}-k^{*}-\gamma}{2}, k^{*}\right) .
$$


Moreover, substituting this value of $k_{1}$ in $\Pi_{i+1}\left(\cdot, k^{*}\right)$, we see that

$$
\Pi_{i^{*}+2}\left(\frac{A_{i^{*}+2}-k^{*}-\gamma}{2}, k^{*}\right)=\Pi_{i^{*}+1}\left(\frac{A_{i^{*}+2}-k^{*}-\gamma}{2}, k^{*}\right) .
$$

Now, by strict concavity of $\Pi_{i^{*}+1}$ and the fact that $\tilde{k}_{11}<\frac{A_{i^{*}+1}-k^{*}-\gamma}{2}$, it follows that

$$
\Pi_{i^{*}+1}\left(\frac{A_{i^{*}+2}-k^{*}-\gamma}{2}, k^{*}\right)<\Pi_{i^{*}+1}\left(\frac{A_{i^{*}+1}-k^{*}-\gamma}{2}, k^{*}\right) .
$$

since $\left(\frac{A_{i^{*}+2}-k^{*}-\gamma}{2}>\frac{A_{i \cdot+1}-k^{*}-\gamma}{2}\right)$. Furthermore, using (A.3) and (A.6), we get

$$
\Pi_{i^{*}+1}\left(\frac{A_{i^{*}+2}-k^{*}-\gamma}{2}, k^{*}\right)<\Pi_{i^{*}}\left(\frac{A_{i^{*}+1}-k^{*}-\gamma}{2}, k^{*}\right) \leq \Pi_{i^{*}}\left(k^{*}, k^{*}\right) .
$$

Finally comparing (A.4) and (A.7), we obtain

$$
\Pi_{i^{*}+2}\left(k_{1}, k^{*}\right) \leq \Pi_{i^{*}}\left(k^{*}, k^{*}\right)
$$

for all $k_{1} \in I_{i^{*}+2}^{*}$, which proves that no deviation $k_{1}$ in $I_{i^{*}+2}^{*}$ is profitable.

Proceeding in the same fashion for $k_{1}$ in $I_{i^{*}+3}, I_{i^{*}+4} \cdots I_{n}$, it can be shown that no deviation $k_{1} \geq k^{*}$ can be profitable for firm 1 .

Now let us consider deviations $k_{1}$ with $k_{1} \leq k^{*}$. When $\frac{A_{i^{*}}-\gamma}{3} \leq k_{1}<k^{*}$, it is clear that such a deviation cannot be profitable, since $k^{*}$ is the best reply in the first stage game against $k^{*}$ as long as $k_{1}$ is in the interval $\left[\frac{A_{i^{*}}-\gamma}{3}, \frac{A_{i^{*}+1}-\gamma}{3}\right]$. So we have only to consider the values of $k_{1} \leq \frac{A_{i}-\gamma}{3}$. Let $s$ be any state with $1 \leq s<i^{*}$. Denote by $D_{s}$ the interval $\left[\frac{A_{s}-\gamma}{3}, \frac{A_{s+1}-\gamma}{3}\left[, 1<s \leq i^{*}-1\right.\right.$. When $k_{1} \in D_{s}, 1<s \leq i^{*}-1$, the payoff $\Pi_{s}\left(k_{1}, k^{*}\right)$ of firm 1 in the first game is given by

$$
\begin{aligned}
\Pi_{s}\left(k_{1}, k^{*}\right) & \stackrel{\text { def }}{=} \sum_{\sigma=1}^{s-1} \rho_{\sigma}\left(\frac{A_{\sigma}-\gamma}{3}\right)^{2}+\sum_{\sigma=s}^{i^{*}} \rho_{\sigma}\left(A_{\sigma}-\frac{A_{\sigma}-\gamma}{3}-k_{1}-\gamma\right) k_{1} \\
& +\sum_{\sigma=i^{*}+1}^{n} \rho_{\sigma}\left(A_{\sigma}-k^{*}-\gamma-k_{1}\right) k_{1}-\beta k_{1} .
\end{aligned}
$$


Assume first that $k_{1} \in D_{i^{*}-1}$. In this domain, the payoff of the first stage game, given that firm 2 plays $k^{*}$, obtains by applying formula (II) with $s=i^{*}-1$, i.e.

$$
\begin{aligned}
\Pi_{i^{*}-1}\left(k_{1}, k^{*}\right) & =\sum_{\sigma=1}^{i^{*}-1} \rho_{\sigma}\left(\frac{A_{\sigma}-\gamma}{3}\right)^{2}+\rho_{i^{*}}\left(A_{i^{*}}-\frac{A_{i^{*}}-\gamma}{3}-k_{1}-\gamma\right) k_{1} \\
& +\sum_{\sigma=i^{*}+1}^{n} \rho_{\sigma}\left(A_{\sigma}-k^{*}-k_{1}-\gamma\right) k_{1}-\beta k_{1} .
\end{aligned}
$$

It is easy to theck that $\frac{\partial^{2} \Pi_{i^{*}-1}}{\partial K_{1}}<0$, so that $\Pi_{i \cdot-1}$ is strictly concave. On the other hand,

$$
\frac{\partial \Pi_{i^{*}-1}}{\partial k_{1}}=0 \Leftrightarrow k_{1}=\tilde{k}_{13} \stackrel{\text { def }}{=}\left[\frac{\sum_{\sigma=i^{*}}^{n} \rho_{\sigma} A_{\sigma}-\beta}{\sum_{\sigma=i^{*}}^{n} \rho_{\sigma}}\right]-\frac{\gamma}{3} .
$$

Notice that if $\tilde{k}_{13}$ is strictly smaller than $\frac{A_{i^{*}}-\gamma}{3}$, then we would have

$$
\beta>\sum_{\sigma=i^{*}+1}^{n} \rho_{\sigma}\left(A_{\sigma}-A_{i^{*}}\right),
$$

which contradicts the right part of inequality (A.1).

Accordingly, the value of $k_{1}$ which maximizes the function $\Pi_{i^{*}-1}$ exceeds the right extreme of the interval $D_{i^{*}-1}$. Thus, by strict concavity of $\Pi_{i^{*}-1}\left(k_{1}, k^{*}\right)$, the optimal value of $\Pi_{i^{*}-1}\left(k_{1}, k^{*}\right)$ when $k_{1}$ is restricted to $D_{i^{*}-1}$, lies on the right boundary of $D_{i^{*}-1}$, i.e. at $k_{1}=\frac{A_{i^{*}}-\gamma}{3}$. Moreover, substituting this value of $k_{1}$ in (2), we see that

$$
\Pi_{i^{*-1}}\left(\frac{A_{i^{*}}-\gamma}{3}, k^{*}\right)=\Pi_{i^{*}}\left(\frac{A_{i^{*}}-\gamma}{3}, k^{*}\right) \leq \Pi_{i^{*}}\left(k^{*}, k^{*}\right) .
$$

Accordingly, no deviation from $k^{*}$ can be profitable by reducing capacity in $D_{i^{*}-1}$. Applying the analogous reasoning held above for values of $k_{1} \leq k^{*}$, it can be shown that no deviation in $D_{i^{*-2}}, D_{i^{*-3}}, \cdots, D_{1}$ can be profitable to firm 1 . Consequently, no deviation $k_{1} \leq k^{*}$ can be profitable for firm 1 . A perfectly similar argument applies to show that no deviation from $k^{*}$ can be profitable either for firm 2.

This completes the proof of proposition 2 . 